Zu diesem Gesamtlösungspaket gehört auch der Support von HP. Das High Availability Observatory ( $\mathrm{HAO}$ ) ist eine Technologielösung, die unternehmenskritische Systembereiche überwacht, Hardware-Probleme erkennt und bei Fehlern selbstständig die Techniker benachrichtigt. Solite etwa eine Festplatte im Cluster der austriamicrosystems AG ausfallen, behebt das HP Support Team dieses Problem umgehend.

\section{Eine komplexe Lösung}

Die Produktion läuft dank der Konzeptarbeit von ASCAD zuverlässig. Gerade bei der austriamicrosystems AG hätte ein längerer IT-Ausfall im Produktionssystem verheerende wirtschaftliche Folgen. Er ist für das Unternehmen gleichsam nicht akzeptabel. Laut Dipl.-ing. Alexander Scheidl, der für die Projektumsetzung bei austriamicrosystems AG verantwortlich ist, macht sich die Einplanung aller Eventualitäten vor der Implementierung eines so hoch komplexen, maßgeschneiderten Systems bezahlt.

\section{ASCAD und HP}

ASCAD ist Spezialist für Hochverfügbarkeit auf allen Betriebssystemen. Das Un- ternehmen mit Sitz in Korneuburg bietet maßgeschneiderte Lösungen in den $\mathrm{Be}$ reichen Storage, Security, Backup, System- und Netzwerkmanagement. Hewlett-Packard ist Anbieter von Produkten, Technologien, Lösungen und Dienstleistungen für Privatkunden und Unternehmen.

Informationen: ASCAD Informationstechnologie Gesellschaft m.b.H., Brigitte Stoimenov, Telefon +43 (2266) 71 600-0,

E-Mail: brigitte.stoimenov@ascad.at,

Internet: www.ascad.at; Hewlett-Packard, Elmar Furtenbach, Telefon +43 (1) 25000-6365 E-Mail: elmar.furtenbach@hp.com,

Internet: www.hp.com/at

\title{
Neue Turbinen für Kraftwerk Agonitz
}

\begin{abstract}
Die VA TECH HYDRO und die Energie AG Oberösterreich weiten ihre Zusammenarbeit im Wasserkraftwerksbau aus und starten ein weiteres gemeinsames Entwicklungsprojekt. Das neue Turbinenkonzept ist eine innovative Anwendung einer bewährten Technologie und darüber hinaus gut geeignet für einen nachträglichen Einbau.
\end{abstract}

Im Energie AG-Kraftwerk Agonitz an der Steyr wird der Prototyp der Straflo-Matrix ${ }^{\mathrm{TM}}$, einer von VA TECH HYDRO weiterentwickelten HYDROMATRIX®-Turbine, für den weltweiten Einbau in bestehende Wehranlagen oder Bewässerungsdämme erprobt.

\section{Die nächste Turbinen- generation}

Das Konzept der HYDROMATRIX-Turbine ist die innovative Anwendung einer bewährten Technologie. In einer Art Baukastensystem ist eine Vielzahl kleiner, identer Turbinengeneratoreinheiten in einem gemeinsamen Rahmen in Matrixform angeordnet.

Die Besonderheit des Straflo-Matrix-Systems, eine Weiterentwicklung der HYDROMATRIX-Turbine, ist die Ausfühtrung des Generators. Das Turbinenlaufrad dient dabei gleichzeitig als Träger des Generatorrotors. Die dadurch resultierende kleinere Bauweise ist noch wirtschaftlicher und hat große Vorteile bei engen Platzverhältnissen.

\section{Nachträglicher Einbau}

Der hohe wirtschaftliche Nutzen der

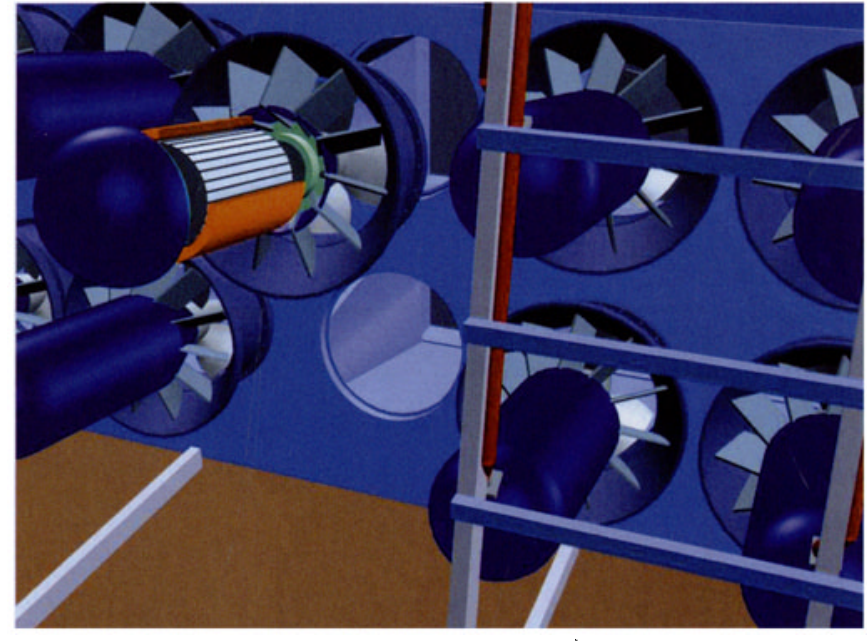

Bei der HYDROMATRIX-Turbine sind mehrere kleine, idente Turbinengeneratoreinheiten in einem gemeinsamen Rahmen in Form einer Matrix angeordnet

3. Die Turbine am Prüfstand

Die Straflo-Matrix-Turbine wurde im Herbst im Energie AG-Kraftwerk Agonitz termingerecht eingebaut und wird nun in einem mehrwöchigen Probebetrieb getestet. Die intensiven und eingehenden Tests sowie die Erkenntnisse aus der Betriebserfahrung liefern einen wichtigen Beitrag zum Erreichen der Serienreife.

Im Jänner sollen die Untersuchungen an der neuen Turbine abgeschlossen sein und mit den Umbauarbeiten am Kraftwerk begonnen werden. Die Energie AG hat das Kraftwerk Agonitz im Jahre 1996 zusammen mit den Kraftwerken Steinbach und

Straflo-Matrix-Turbine ist so wie bei der HYDROMATRIX-Turbine dadurch gegeben, dass diese in bereits bestehende Bauwerke wie Wehr- und Schleusenanlagen oder Bewässerungssysteme auch nachträglich eingebaut werden kann. Daraus eröffnen sich weltweit neue Chancen, bisher ungenutzte Wasserkraftpotenziale aufgrund der geringen Investitionskosten wirtschaftlich $\mathrm{zu}$ realisieren.
Humpelmühle aus privater Hand übernommen. Nach dem Umbau des Kraftwerks wird hier mit Jahreswechsel 2003/2004 die weltweit erste kommerziell genutzte Straflo-Matrix-Turbine zum Einsatz kommen.

Informationen: VA TECH HYDRO GmbH \& Co, Dr. Ursula Scheidl, Penzinger Straße 76, A-1140 Wien, Telefon +43 (1) 89 100-2053,

E-Mail: ursula.scheidl@vatech-pw.com, Internet: www.vatech-hydro.com 